\title{
Cosmology with galaxy clusters: studying the Dark Ages and the Epoch of Reionization in the SKA era
}

\author{
Sergio Colafrancesco*1, Paolo Marchegiani ${ }^{1}$, M. Shehzad Emritte ${ }^{1}$ \\ ${ }^{1}$ School of Physics, University of the Witwatersrand, 1 Jan Smuts Ave, WITS-2050 \\ Johannesburg, South Africa \\ E-mail: sergio.colafrancescodwits.ac.za
}

\begin{abstract}
The cosmological evolution during the Dark Ages (DA) and the Epoch of Reionization (EoR) marks an important transition in the history of the universe that it is not yet fully understood. We study here a new technique to probe the history and the physics of the DA and EoR which makes use of the Comptonization of the cosmic microwave background (CMB) spectrum, modified by the physical effects occurring during this epoch related to the $21-\mathrm{cm}$ radiation background, as induced by thermal and non-thermal electrons residing in the atmospheres of cosmic structures like galaxy clusters, radiogalaxy lobes and galaxy halos: we refer to this effect as SZE- $21 \mathrm{~cm}$. The spectral features of the thermal and non-thermal SZE- $21 \mathrm{~cm}$ depend on the history and on the physical mechanisms occurring during the DA and EoR. We find that the redshift location of the main physical mechanisms occurring during the DA and EoR leave an imprint on the spectral shape of the SZE-21 cm that is observable mainly in the 70-350 MHz range. We also prove that two different kinds of SZE-21 cm are observable, the thermal and non-thermal SZE- $21 \mathrm{~cm}$, and have different spectral shapes and frequency location, depending on the gas temperature (for the thermal SZE-21 cm) and electrons minimum momentum (for the non-thermal SZE-21 cm). The spectral imprint of the DA and EoR is maximized for clusters with high plasma temperature and optical depth, thus making rich clusters the optimal laboratories to study the $S Z E-21 \mathrm{~cm}$. We show that the global SZE-21 cm signal can be detected by SKA1-LOW in the range $v \gtrsim 70-90 \mathrm{MHz}$ for clusters with temperature $\gtrsim 20-5 \mathrm{keV}$ respectively, and that we can separate the SZE- $21 \mathrm{~cm}$ from the standard SZE with SKA2 at $v \sim 100 \mathrm{MHz}$ for clusters with temperature $\gtrsim 15 \mathrm{keV}$.
\end{abstract}

Advancing Astrophysics with the Square Kilometre Array,

June 8-13, 2014

Giardini Naxos, Sicily, Italy

* Speaker. 


\section{Probing the Dark Ages with galaxy clusters}

Clusters of galaxies have been recognized since several decades as powerful cosmological tools and invaluable astrophysical laboratories (see, e.g., Kravtsov \& Borgani 2012, Colafrancesco 2010). We will discuss here the role of the SKA to provide another fascinating possibility in using galaxy clusters as direct probes of the Dark Ages (DA) and of the Epoch or Reionization (EoR). The most promising method to study the content of the universe during the DA and the beginning of the EoR (see, e.g., Koopmans 2015) is to search for signatures of the (highly redshifted) 21-cm hyperfine transition line of neutral hydrogen in the radio background spectrum (Pritchard \& Loeb 2012). The 21-cm signal from the DA appears as a faint, diffuse background detectable at radio frequencies below $200 \mathrm{MHz}$ (for redshift $z>6$ ). Great opportunities for studying the EoR and the DA through the observations of the 21-cm background are offered by the SKA (Koopmans 2015). During the DA and EoR the CMB radiation spectrum is modified by various physical mechanisms. Subsequent to recombination, the temperature of neutral gas is coupled to that of the CMB, and no changes in the CMB spectrum can be observed. At redshifts below $\approx 200$ the gas cools adiabatically, its temperature drops below that of the CMB, and neutral Hydrogen resonantly absorbs CMB flux through the spin-flip transition (Field 1959, Scott \& Rees 1990, Loeb \& Zaldarriaga 2004). Heating effects of the neutral gas may also occur at high $z$. As the first Dark Matter (DM) clumps form in the universe, the WIMP DM annihilation can then produce a substantial heating of the surrounding IGM (Valdes et al. 2013). At much lower redshifts $z \lesssim 20$, gas temperature is also expected to heat up again as luminous sources turn on and their UV and soft X-ray photons reionize and heat the gas (Chen \& Miralda-Escude 2004). An additional spectral signature is also expected from the Lyman- $\alpha$ radiation field produced by first sources (Barkana \& Loeb 2005) through the Wouthuysen-Field effect (Wouthuysen 1952, Field 1959).

Such CMB spectral distortions offer an alternative and/or complementary method to study the DA and EoR by using the modification induced by the inverse Compton scattering of the CMB photon background, modified by the cosmic $21-\mathrm{cm}$ background frequency spectrum, on intervening electrons in the atmospheres of various cosmic structures, like galaxy clusters, radiogalaxy lobes and galactic halos. We refer here to this effect as the SZE-21cm (Colafrancesco \& Marchegiani 2014). Observations of the SZE- $21 \mathrm{~cm}$ can be carried out with interferometers since the modification associated with low-redshift scattering can be established from differential observations towards and away from galaxy clusters and other cosmic structures containing diffuse thermal and non-thermal plasmas. Unlike an experiment to directly establish the cosmic $21-\mathrm{cm}$ frequency spectrum at low radio frequencies involving a total intensity measurement on the sky, differential observations with radio interferometers are less affected by the exact calibration of the observed intensity using an external source and by the confusion from Galactic foregrounds that are uniform over angular scales larger than those of a typical cluster, as the Galactic synchrotron background at low radio frequencies. Also, since the SZE does not depend on the redshift of the scattering cloud (e.g. clusters, radiogalaxy lobes), it is more suitable to study sources located at high- $z$, allowing to reduce the importance of the intrinsic radio emission of the source with respect to the SZE, and hence allowing to detect a larger number of sources, thus increasing the possibility to obtain more precise results by studying this effect in many sources at cosmological scales.

For this purpose, it is crucial to study the properties of the SZE- $21 \mathrm{~cm}$ in detail, by using a full 
relativistic formalism suitable to study the SZE in a wide range of cosmic structures, including high-temperature gas in galaxy clusters as well as non-thermal and relativistic plasmas therein contained or associated with other cosmic structures like radio galaxy lobes (Colafrancesco et al. 2003, Colafrancesco 2008, Colafrancesco \& Marchegiani 2014). Our general and complete approach is hence necessary to replace the simple and incorrect description of the SZE- $21 \mathrm{~cm}$ provided by Cooray (2006) that is performed in a non-relativistic approximation, considers a single thermal electron population and a single benchmark model for the CMB modified by the $21-\mathrm{cm}$ background, and yields estimates of the SZE- $21 \mathrm{~cm}$ which are a factor from $\approx 40 \%$ to $\gtrsim 3$ in disagreement with the correct calculation at the frequencies where the effect can be observed (Colafrancesco \& Marchegiani 2014). The approach we present here provides a correct estimate of the SZE-21 cm signals in various cosmic structures and thus allows to increase the number of objects that can be studied in this framework, including galaxy clusters with different properties, as radio halos, cooling flows and complex morphologies, and other extragalactic sources as radio galaxies. Moreover, in order to have a complete description of the CMB spectrum modified by the $21-\mathrm{cm}$ background, we will also discuss models that include the effects of the DM annihilation heating.

\section{The SZE-21cm}

The input radiation field used here to calculate the SZE- $21 \mathrm{~cm}$ considers a general model taking into account three main sources: i) absorption and collision effects at $z \sim 200-30$; ii) Ly- $\alpha$ radiation field at $z \sim 30-20$, and iii) X-ray photon heating at $z \sim 20-6$ (details are given in Colafrancesco \& Marchegiani 2014, Cooray 2006). We use this modified CMB radiation field scheme as a benchmark case for the sake of a general discussion of the SZE- $21 \mathrm{~cm}$ and its ability to probe the DA and EoR. We then use the results of the $21 \mathrm{~cm}$-fast code, with the addition of DM annihilation heating effects (Evoli and Ferrara, private communication, Valdes et al. 2013), in order to explore more detailed physical effects occurring during the DA and EoR. The modified CMB spectrum due to the $21-\mathrm{cm}$ radiation, for both the benchmark case and the specific models of Valdes et al. (2013), is shown in Fig.1. The modified CMB spectrum emerging from the DA and EoR (see Fig.1) is then scattered by electrons (thermal and non-thermal) residing in the atmospheres of various cosmic structures (e.g., galaxy clusters, radiogalaxy lobes, galactic halos) and can be then measured by using a differential observing strategy on and off the specific cosmic structure and produce the specific SZE-21 cm we discuss here. The spectral distortion due to the SZE of the $\mathrm{CMB}$ that is modified and emerged from the DA and EoR is given by

$$
I_{\text {mod }}(x)=\int_{-\infty}^{+\infty} I_{0, \bmod }\left(x e^{-s}\right) P(s) d s
$$

(see Colafrancesco et al. 2003, Colafrancesco \& Marchegiani 2014 for a general derivation), where $x=h v /\left(k_{B} T_{0}\right)$ is the normalized photon frequency, $T_{0}$ is the CMB temperature, $h$ in the Planck constant and $k_{B}$ the Boltzmann constant, $P(s)$ is the photon redistribution function (yielding the probability of a logarithmic shift $s=\ln \left(v^{\prime} / v\right)$ in the photon frequency) that depends on the electrons spectrum producing the CMB Comptonization, and $I_{0, \bmod }(x)$ is the specific intensity of the incident CMB radiation field as modified during the DA and EoR. The function $P(s)$ can be calculated at the desired approximation order in the plasma optical depth $\tau$ or via a general relativistic 

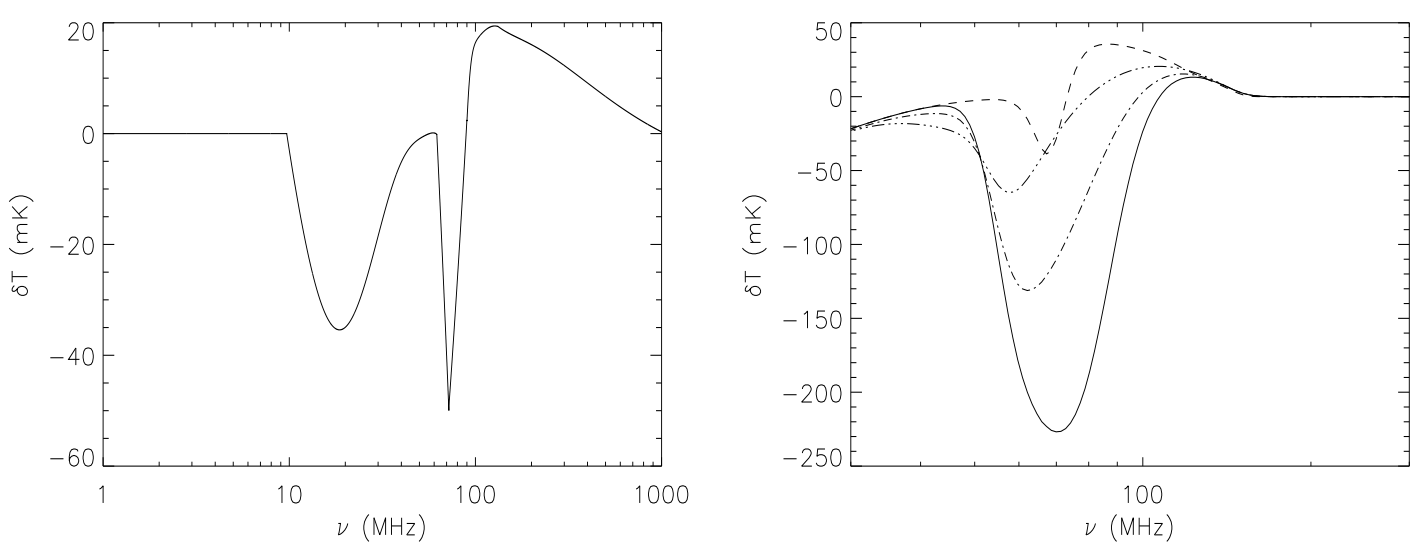

Figure 1: Modified CMB spectrum due to the 21-cm radiation (in units of brightness temperature relative to the CMB) for the benchmark case (left, from Cooray 2006), and for the models with DM heating (right, from Valdes et al. 2013). The right panel shows a fiducial model without DM (solid line), an extreme model without DM (dashed line), a fiducial model with DM with $M_{\min }=10^{-3} \mathrm{M}_{\odot}$ (dot-dashed line), where $M_{\min }$ is the mass of the smallest DM subhalo, and a fiducial model with DM with $M_{\min }=10^{-6} \mathrm{M}_{\odot}$ (three dots-dashed line).

method by using Fourier transform properties (see Colafrancesco et al. 2003 for details). Once the Comptonized spectrum in eq.(2.1) is calculated, the SZE is given by the difference:

$$
\Delta I_{\text {mod }}(x)=I_{\text {mod }}(x)-I_{0, \bmod }(x),
$$

where $I_{0, \bmod }(x)$, written as a function of the frequency $v$, is given by

$$
I_{0, \text { mod }}(v)=I_{0}(v)+\delta I(v) .
$$

The modification to the CMB spectrum, $\delta I(v)$, can be expressed in terms of brightness temperature change relative to the $\mathrm{CMB}$, defined as:

$$
\delta T(v)=\frac{c^{2}}{2 k_{B} v^{2}} \delta I(v)
$$

In the following we discuss the results obtained for the specific case of the SZE- $21 \mathrm{~cm}$ produced by thermal electron populations, that provide the dominant contribution to the SZE observed in galaxy clusters, and by non-thermal electrons populations, that are present in clusters that show non-thermal activity (i.e. radio halos or relics) and in the extended lobes of radiogalaxies. We find that: $i)$ the variations of the SZE- $21 \mathrm{~cm}$ w.r.t. the standard SZE with the non-modified CMB spectrum are more relevant for high-T clusters and for non-thermal electron plasmas with high values of minimum momentum $p_{1}$ (see Fig.2); ii) studying the spectrum of the SZE-21 cm allows to derive precise information on the epoch at which the CMB has been modified and on the physical mechanism that produced such modifications. Specifically, the negative peak at $\sim 60 \mathrm{MHz}$ reflects the transition epoch between the collision/absorption and the Lyman- $\alpha$ interactions (at $z \sim 20-30$ ), while the positive peak at $\sim 70 \mathrm{MHz}$ reflects the effect of the Lyman- $\alpha$ deep absorption in the CMB spectrum $(z \sim 20)$, and the negative peak at $\sim 100 \mathrm{MHz}$ shows the effect of the X-rays and UV reionization produced by first bright sources $(z<20)$. These spectral features in the SZE- $21 \mathrm{~cm}$ 

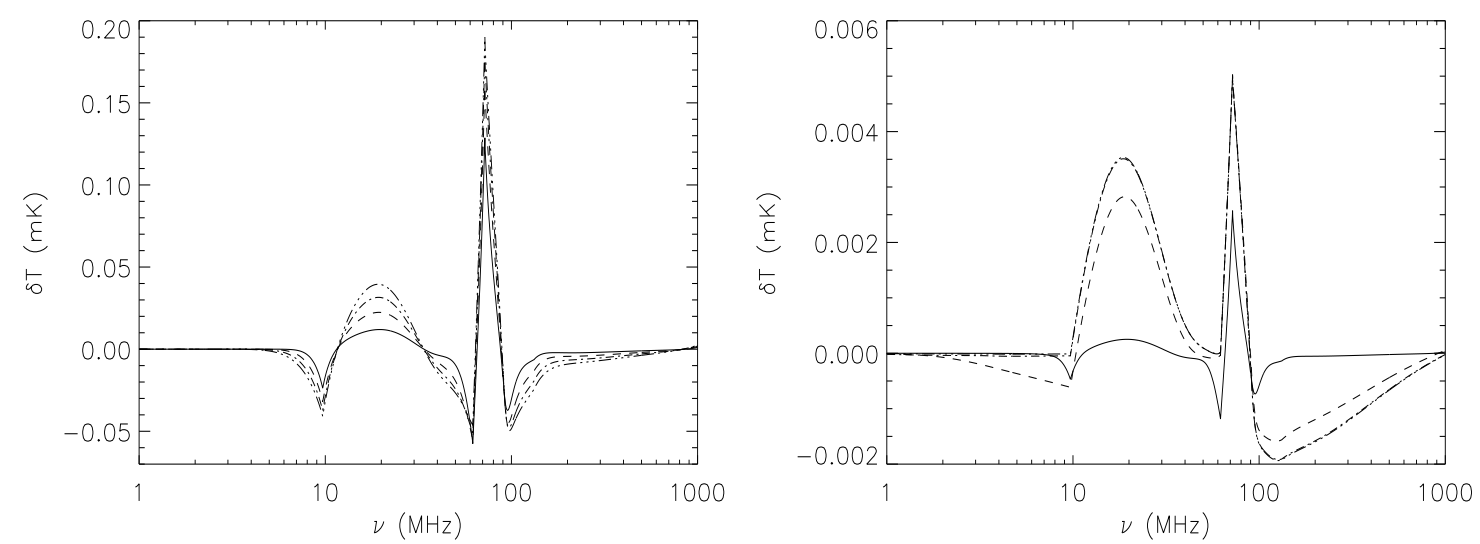

Figure 2: Left. Difference between the SZE-21 cm and the standard SZE (in units of brightness temperature relative to the $\mathrm{CMB}$ ) for clusters with thermal plasma at temperature $k_{B} T=5,10,15$ and $20 \mathrm{keV}$ as shown by the solid, dashed, dot-dashed and dash-three dots lines, respectively. A constant value of the thermal electrons optical depth, $\tau=5 \times 10^{-3}$, has been used in the calculations. Right. Difference between the SZE- $21 \mathrm{~cm}$ and the standard SZE (in units of brightness temperature relative to the CMB) for non-thermal electrons with a power-law spectrum with spectral index $s=3.5$ and minimum momentum $p_{1}=0.1,1,5$ and 10, shown by the solid, dashed, dot-dashed and dash-three dots lines, respectively. A constant value of the non-thermal electrons optical depth, $\tau=1 \times 10^{-4}$, has been used in the calculations.
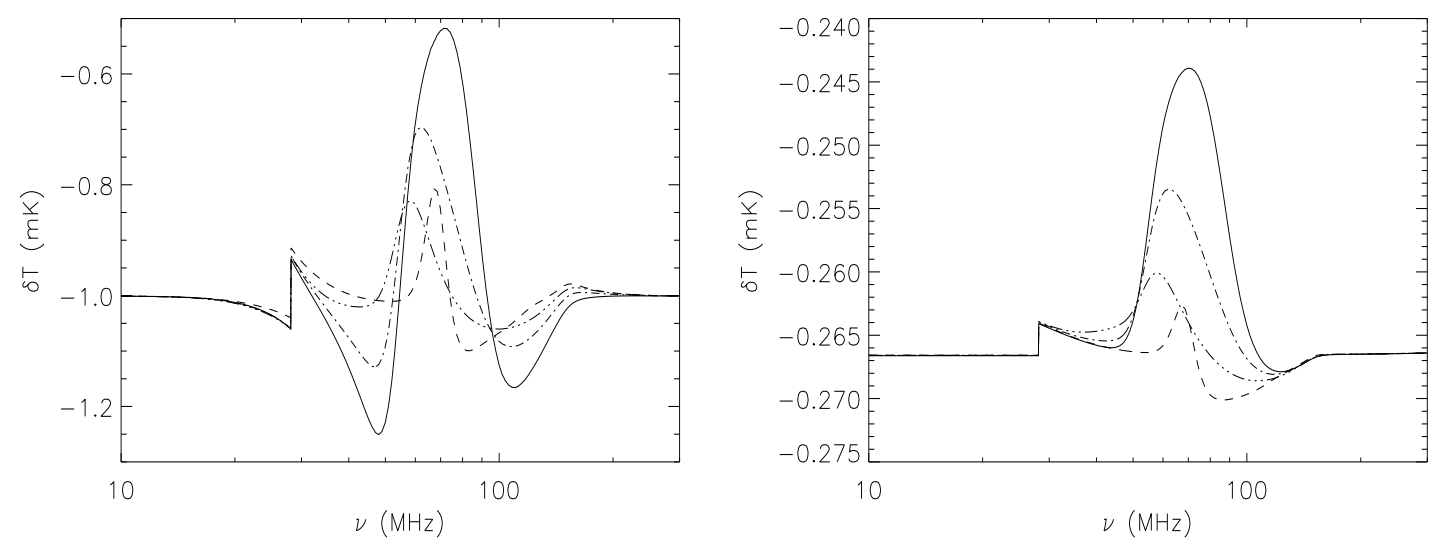

Figure 3: The SZE-21cm (in units of brightness temperature relative to the $\mathrm{CMB}$ ) for a thermal plasma with $k_{B} T=20$ $\mathrm{keV}$ and $\tau=5 \times 10^{-3}$ (left panel) and for a non-thermal plasma with $s=3.5$ and $p_{1}=10$ and with $\tau=1 \times 10^{-4}$ (right panel). Both figures are calculated using the models of Valdes et al. (2013) for a modified CMB spectrum with a fiducial model without DM (solid line), an extreme model without DM (dashed line), a fiducial DM model with $M_{\min }=10^{-3}$ $\mathrm{M}_{\odot}$ (dot-dashed line), and a fiducial DM model with $M_{\min }=10^{-6} \mathrm{M}_{\odot}$ (three dots-dashed line).

reflect hence the frequency location (i.e., the redshift occurrence) of the three main physical effects modifying the CMB spectrum. The SZE- $21 \mathrm{~cm}$ calculated with the specific models in Fig.3 shows similar features in respect to the benchmark case, especially for the fiducial model without DM, where similar assumptions are made. In the extreme model, the effect of reionization by astrophysical sources is stronger, and this produces a damping in the modification to the CMB spectrum and to the SZE- $21 \mathrm{~cm}$. Similar effects can be produced also by DM annihilation heating, and in this case the damping is stronger in models with smaller values of minimum DM halo mass, since the 
effect of smaller halos is more effective in heating the gas (Valdes et al. 2013); iii) the thermal and non-thermal SZE- $21 \mathrm{~cm}$ have quite different spectral shapes. Thus, it is possible, in principle, to derive information also on the existence and the properties of the electron populations in cosmic structures also from low- $v$ observations of the SZE- $21 \mathrm{~cm}$. This property is complementary with the results of previous studies, according to which the properties of non-thermal electrons can be derived from the study of the SZE at higher frequencies (see, e.g., Colafrancesco et al. 2011, Colafrancesco 2013).

\section{SKA observations of the SZE-21cm}

Our analysis shows that most of information on the measurements of the SZE-21cm will come from the SKA1-LOW, for $v \lesssim 300 \mathrm{MHz}$ and additional/complementary information can also be obtained from SKA1-MID. The range of frequencies at which the SZE- $21 \mathrm{~cm}$ can be observed is from 10 to $300 \mathrm{MHz}$ and the SKA1-LOW band 50-350 MHz is optimally suited to observe the thermal SZE-21 cm. The SKA1-MID Band $1(350-1050 \mathrm{MHz})$ is also interesting to address the observability of the tail in the non-thermal SZE- $21 \mathrm{~cm}$. In Figure 4 we compare the flux calculated for the modified $\mathrm{CMB}$ and the one calculated for the non-modified CMB with the sensitivity of SKA1-LOW for $100 \mathrm{kHz}$ bandwith, $1000 \mathrm{hrs}$ of integration, 2 polarizations, no taper, no weight, and with the sensitivities of SKA-50\% and SKA2 in the same conditions, by assuming a collecting area respectively $50 \%$ and $400 \%$ than SKA1. We find that the SZE- $21 \mathrm{~cm}$ can be detected with SKA1 at frequencies $v \sim 70 \mathrm{MHz}$ for clusters with temperature $k T \approx 20 \mathrm{keV}$ and at $v \sim 90 \mathrm{MHz}$ even for relatively low temperature clusters $k T \approx 5 \mathrm{keV}$.

Disentangling between the SZE-21 cm and the standard SZE signals is more difficult: the difference between the two signals is always at most of the order of few $\mu \mathrm{Jy}$ (see Figure 4, right panel), so this requires to measure the signal with high precision and at frequencies where the differences are larger. Good frequency channels for this purpose can be found at $62 \mathrm{MHz}$ (where the SZE$21 \mathrm{~cm}$ is stronger than the standard SZE because of the transition between collisions effect and Lyman- $\alpha$ interactions), $72 \mathrm{MHz}$ (where the SZE- $21 \mathrm{~cm}$ is lower because of the Lyman- $\alpha$ peak), and at $96 \mathrm{MHz}$ (where the SZE- $21 \mathrm{~cm}$ is stronger because of the X-ray heating effect). Because of the better sensitivity of SKA1-LOW at high frequencies, the best frequency range where we can obtain information on the SZE- $21 \mathrm{~cm}$ is $v \geq 96 \mathrm{MHz}$. However, also in this frequency range the difference between the two signals is of the order of $\mu \mathrm{Jy}$, so very deep observations, and very accurate data analysis are required, together with the use of clusters with high values of temperature and optical depth. An appropriate strategy could be to select the SZE clusters at higher (mm. and sub-mm.) frequencies to derive precise information on the parameters of the ICM, and then use these constraints to obtain a better estimate of the properties of the SZE-21 cm with SKA1-LOW. Instead, the goal to detect the difference between the standard and $21 \mathrm{~cm}-S Z E$ can be achieved more easily with SKA2, for integration times of $1000 \mathrm{hrs}$. The detection of the non-thermal SZE- $21 \mathrm{~cm}$ in e.g. radiogalaxy lobes or cluster radio halos is more challenging being this signal a factor $\sim 40$ or more lower than the thermal SZE- $21 \mathrm{~cm}$. However, the different spectral features of the non-thermal and thermal SZE-21cm (see Fig.2) can allow, in principle, a detection of this signal and hence an estimate of non-thermal cluster or radiogalaxy lobe properties independently of measurements in other spectral bands. In fact, it is possible to strategize the search of this signal in objects where 

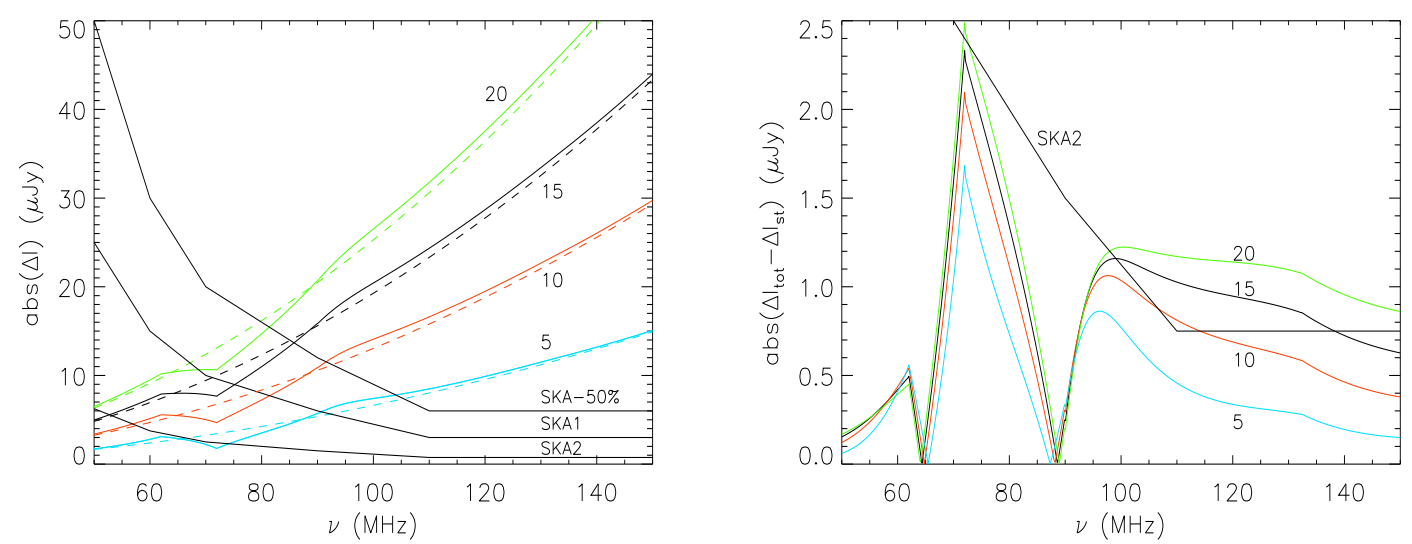

Figure 4: Left: spectra of the fluxes of the SZE-21 cm (in units of $\mu \mathrm{Jy}$ and in absolute value with the solid lines) and the SZE for a non-modified CMB (dashed lines) compared with the SKA1-LOW sensitivity for $100 \mathrm{kHz}$ bandwith, 1000 hrs of integration, 2 polarizations, no taper, no weight, and the corresponding sensitivities for SKA-50\% and SKA2 (thick lines). Right: absolute value of the difference between the SZE- $21 \mathrm{~cm}$ and the standard SZE for a non-modified CMB compared with the SKA2 sensitivity for $100 \mathrm{kHz}$ bandwith, $1000 \mathrm{hrs}$ of integration (thick line), 2 polarizations, no taper, no weight. Both figures refer to thermal plasma with temperatures $k_{B} T=20$ (green), 15 (black), 10 (red) and 5 (cyan) $\mathrm{keV}$, and are calculated for $\tau_{0}=5 \times 10^{-3}$, and a spatial profile given by a beta model with $\theta_{c}=300 \operatorname{arcsec}, \beta=0.75$, $\theta_{\max }=10 \theta_{c}$ (see Colafrancesco \& Marchegiani 2014 for details).

the non-thermal components are dominant, such as in the case of radio galaxies lobes with more energetic electrons (i.e. with harder radio spectra), large optical depth (for which a good indication could be a strong radio luminosity) and high redshift, and to adopt a stacking analysis to derive information on the non-thermal SZE- $21 \mathrm{~cm}$.

We stress here that the probe of the DA and EoR with the SZE- $21 \mathrm{~cm}$ technique we propose here would necessitate, in principle, of only one very deep observation of a hot massive cluster and one observation of a high optical depth radiogalaxy lobe, because the spectral features of the SZE$21 \mathrm{~cm}$ are unique and not depending on the redshift of the source. However, observing a sample of galaxy clusters in the temperature range $10-20 \mathrm{keV}$ and a sample of radiogalaxy lobes with various spectra will provide a better characterization of the SZE- $21 \mathrm{~cm}$ spectral shape and hence a more precise determination of the DA and EoR spectral (redshift) features. A possible observational strategy for studying the SZE-21cm with the SKA is to observe $i$ ) a sample of high temperature and optical depth clusters in the souther hemisphere with high values of the Compton parameter $Y_{S Z} \propto \int d \ell P_{e}$ from Planck, SPT and ACT SZE source catalogs, and ii) a sample of radiogalaxies with extended lobes from existing catalogs of giant radio galaxies (GRG).

The range of frequencies at which the SZE-21cm can be observed is from 10 to $300 \mathrm{MHz}$ and the SKA1-LOW band $50-350 \mathrm{MHz}$ is optimally suited to observe the thermal SZE- $21 \mathrm{~cm}$. The SKA1-MID Band $1(350-1050 \mathrm{MHz})$ is also interesting to address the observability of the tail in the non-thermal SZE- $21 \mathrm{~cm}$.

The reference resolution of SKA1-LOW at $110 \mathrm{MHz}$, corresponding to a minimum baseline of 50 $\mathrm{km}$, is $\theta_{\min } \sim 11$ arcsec. For an isothermal galaxy cluster, with a gas density profile given by a $\beta$-profile (Cavaliere \& Fusco-Femiano 1976), the optical depth at a projected distance $\theta$ from the 
center of the cluster is given by the expression:

$$
\tau(\theta)=\tau_{0}\left[1+\left(\frac{\theta}{\theta_{c}}\right)^{2}\right]^{\frac{1}{2}-\frac{3}{2} \beta}
$$

(Colafrancesco et al. 2003). By assuming values typical of a near and rich galaxy cluster like Coma, i.e. $\tau_{0}=5 \times 10^{-3}, \beta=0.75, \theta_{c}=300 \mathrm{arcsec}$, we can calculate the flux up to $\theta_{\max }=10 \theta_{c}$, and we can estimate that the lack of sensitivity to scales $\theta<\theta_{\min }$ implies a relative signal loss of the order of $\sim 1.1 \times 10^{-4}$ (Colafrancesco \& Marchegiani 2014). Since the signal we aim to detect is of the order of tens of $\mu \mathrm{Jy}$ at these frequencies (see Fig.4), the loss of signal is of the order of $\sim$ $\mathrm{nJy}$, so it does not affect our results.

We note that the differential observations of the SZE- $21 \mathrm{~cm}$ are less affected by issues such as the exact calibration of the observed intensity using an external source and the confusion from galactic foregrounds that are uniform over angular scales larger than a typical cluster, as the Galactic synchrotron background at low radio frequencies. In addition, since the SZE does not depend on redshift, this technique is more suitable to study sources located at relatively large distances, allowing also to reduce the importance of their intrinsic radio emission with respect to the SZE- $21 \mathrm{~cm}$, and allowing hence to detect a larger number of sources, thus increasing the possibility to obtain more precise results by studying this effect in many sources at cosmological scales. We also emphasize here that a particular attention must be dedicated to the subtraction of extended clumps of low- $v$ radio emission affecting the SKA1-LOW band.

Figure 4 shows that an early science study of the SZE- $21 \mathrm{~cm}$ with a $50 \%$ SKA1 performance can be obtained with a limited sample of very high-T clusters that maximize the probability of the SZE$21 \mathrm{~cm}$ detection by producing the largest signals in the SKA1-LOW band. This project will benefit from the expected increase in sensitivity of SKA2 (see Fig.4), but will not depend on the SKA2 frequency coverage extension because the optimal $v$ range is still around $v \gtrsim 100 \mathrm{MHz}$.

\section{Conclusions and future outline}

The SKA offers the unique possibility to probe the DA and the EoR also by using local structures as probes of this remote epoch. The SZE- $21 \mathrm{~cm}$ has the potential to provide precise studies of the DA and EoR, and its detection with the coming SKA can yield unique information on the history and physical mechanisms occurring during the DA and EoR. One of the advantages of using the SZE- $21 \mathrm{~cm}$ is the nature of its differential measurement and our knowledge of the cluster medium from local observations in the X-rays, radio and $\mathrm{mm}$. frequency ranges. Observations have to be carried out towards high-temperature and high-optical depth (i.e., high Compton parameter $Y_{S Z}$ ) clusters to maximize both the amplitude of the signal and the spectral difference between the SZE- $21 \mathrm{~cm}$ and the standard SZE. The best frequency range of observations is between $\approx 70$ and $110 \mathrm{MHz}$, where the difference between the SZE-21 cm and the standard SZE is maximum, and the sensitivity of SKA1-LOW is sufficient to detect the overall signal with $\approx 1000$ hours of integration. Together with such deep observations, a very accurate theoretical analysis is required, where the full formalism to calculate the SZE- $21 \mathrm{~cm}$ and detailed models for the cosmological 21$\mathrm{cm}$ background must be used. It is also important to perform a detailed study of the SZE at higher 
frequencies from existing (e.g., SPT, ACT) and future (e.g., Millimetron, SPT-3G) cluster observations in order to estimate the cluster plasma parameters to be used as prior constraints for the study of the SZE- $21 \mathrm{~cm}$ at the low radio frequencies covered by the SKA. Finally, the independence of the SZE from the redshift of the source allows the study of the SZE- $21 \mathrm{~cm}$ in a larger number of objects distributed over a wider redshift range, therefore producing statistical studies aimed at maximizing the detectable signal that will allow hence a better understanding of the cosmic history of the physical processes occurring during the DA and the EoR.

\section{References}

Barkana, R. and A. Loeb, 2005, Astrophys. J. 626, 1

Cavaliere, A. \& Fusco-Femiano, R, 1976, A\&A, 49, 137

Chen, X.L. and J. Miralda-Escude, 2004, Astrophys. J. 602, 1

Colafrancesco, S., 2008, MNRAS, 385, 2041

Colafrancesco, S. 2010, MmSAIt, 81, 104

Colafrancesco, S. 2013, Acta Pol., 53, 560

Colafrancesco, S., Marchegiani, P. and Palladino, E., 2003, A\&A, 397, 27-52

Colafrancesco, S., Marchegiani, P. \& Buonanno, R., 2011, A\&A, 527, L1

Colafrancesco, S. and Marchegiani, P. 2014, A\&A, submitted

Cooray, A., 2006, Phys. Rev. D, 73, 103001

de Oliveira-Costa, A. et al., 2008, MNRAS, 388, 247

Field, G.B. 1959, Astrophys. J. 129, 536

Koopmans, L. 2015, in proceedings of "Advancing Astrophysics with the Square Kilometre Array", PoS(AASKA14)001

Kravtsov, A.V. and Borgani, S., 2012, ARA\&A, 50, 353

Loeb, A. and Zaldarriaga, M. 2004, Phys. Rev. Lett. 92, 211301

Paciga, G. et al., 2013, MNRAS, 433, 639

Pritchard, J.R., \& Loeb, A. 2012, Rep. Progr. Phys., 75, 086901

Scott, D. and Rees, M.J. 1990, Mon. Not. R. Astr. Soc. 247, 510

Valdes, M., et al., 2013, MNRAS, 429, 1705

Wouthuysen, S.A., 1952, Astron. J., 57, 31 